\title{
A Novel Survival-Based Tissue Microarray of Pancreatic Cancer Validates MUC1 and Mesothelin as Biomarkers
}

\author{
Jordan M. Winter ${ }^{1}$, Laura H. Tang ${ }^{2}$, David S. Klimstra ${ }^{2}$, Murray F. Brennan ${ }^{3}$, Jonathan R. Brody ${ }^{1}$, \\ Flavio G. Rocha ${ }^{4}$, Xiaoyu Jia ${ }^{5}$, Li-Xuan Qin ${ }^{5}$, Michael I. D'Angelica ${ }^{3}$, Ronald P. DeMatteo ${ }^{3}$, Yuman Fong ${ }^{3}$, \\ William R. Jarnagin ${ }^{4}$, Eileen M. O'Reilly ${ }^{6}$, Peter J. Allen ${ }^{5 *}$
}

1 Department of Surgery, Thomas Jefferson University, Philadelphia, Pennsylvania, United States of America, 2 Department of Pathology, Memorial Sloan-Kettering Cancer Center, New York, New York, United States of America, 3 Department of Surgery, Memorial Sloan-Kettering Cancer Center, New York, New York, United States of America, 4 Department of Surgery, Virginia Mason Medical Center, Seattle, Washington, United States of America, 5 Department of Epidemiology and Biostatistics, Memorial SloanKettering Cancer Center, New York, New York, United States of America, 6 Department of Medicine, Memorial Sloan-Kettering Cancer Center, New York, New York, United States of America

\begin{abstract}
Background: One-fifth of patients with seemingly 'curable' pancreatic ductal adenocarcinoma (PDA) experience an early recurrence and death, receiving no definable benefit from a major operation. Some patients with advanced stage tumors are deemed 'unresectable' by conventional staging criteria (e.g. liver metastasis), yet progress slowly. Effective biomarkers that stratify PDA based on biologic behavior are needed. To help researchers sort through the maze of biomarker data, a compendium of $\sim 2500$ published candidate biomarkers in PDA was compiled (PLoS Med, 2009. 6(4) p. e1000046).

Methods and Findings: Building on this compendium, we constructed a survival tissue microarray (termed s-TMA) comprised of short-term (cancer-specific death $<12$ months, $n=58$ ) and long-term survivors $(>30$ months, $n=79)$ who underwent resection for PDA (total, $n=137$ ). The s-TMA functions as a biological filter to identify bona fide prognostic markers associated with survival group extremes (at least 18 months separate survival groups). Based on a stringent selection process, 13 putative PDA biomarkers were identified from the public biomarker repository. Candidates were tested against the s-TMA by immunohistochemistry to identify the best markers of tumor biology. In a multivariate model, MUC1 (odds ratio, $O R=28.95,3+$ vs. negative expression, $p=0.004$ ) and MSLN (OR $=12.47,3+$ vs. negative expression, $p=0.01$ ) were highly predictive of early cancer-specific death. By comparison, pathologic factors (size, lymph node metastases, resection margin status, and grade) had ORs below three, and none reached statistical significance. ROC curves were used to compare the four pathologic prognostic features $(R O C$ area $=0.70)$ to three univariate molecular predictors $(M U C 1, M S L N$, MUC2) of survival group (ROC area $=0.80, p=0.07$ ).

Conclusions: MUC1 and MSLN were superior to pathologic features and other putative biomarkers as predicting survival group. Molecular assays comparing cancers from short and long survivors are an effective strategy to screen biomarkers and prioritize candidate cancer genes for diagnostic and therapeutic studies.
\end{abstract}

Citation: Winter JM, Tang LH, Klimstra DS, Brennan MF, Brody JR, et al. (2012) A Novel Survival-Based Tissue Microarray of Pancreatic Cancer Validates MUC1 and Mesothelin as Biomarkers. PLoS ONE 7(7): e40157. doi:10.1371/journal.pone.0040157

Editor: Frederic Andre, Aix-Marseille University, France

Received March 14, 2012; Accepted June 1, 2012; Published July 6, 2012

Copyright: (C) 2012 Winter et al. This is an open-access article distributed under the terms of the Creative Commons Attribution License, which permits unrestricted use, distribution, and reproduction in any medium, provided the original author and source are credited.

Funding: Funds for the study were derived from U01 CA152653, PI Allen. In addition, funds were provided by the Marshall and Therese Sonenshine Foundation. The funders had no role in study design, data collection and analysis, decision to publish, or preparation of the manuscript.

Competing Interests: The authors have declared that no competing interests exist.

* E-mail: allenp@mskcc.org

\section{Introduction}

While pancreatic ductal adenocarcinoma (PDA) is typically aggressive as compared to most other cancers, the disease is comprised of a range of biological phenotypes. Roughly $20 \%$ of patients who undergo resection will live at least 5 years, and a similar percentage of patients will recur early after resection and die of disease within a year [1-4]. At the genomic level, each PDA acquires a unique constellation of somatic mutations [5]. Molecular diversity at the RNA and protein levels is even more complex. Despite the genotypic and phenotypic diversity in PDA, there are no reliable or clinically relevant prognostic biomarkers that stratify the disease based on predicted outcome.
Pathology reports include basic information regarding the stage and grade of the tumor, and currently provide the best available prognostic information. Conventional pathologic features remain the prognostic gold standard (e.g. lymph node status and histologic grade). However, across multiple large studies, adjusted hazard ratios for pathologic features are below two [6-8]. Similarly, in a validated pancreatic cancer nomogram, adverse pathologic features contribute less than $10 \%$ to 3 -year survival predictions [9]. Serum CA19-9 is equally limited as a prognostic marker [1012]. Prognostic information with such minimal predictive value cannot reliably inform treatment decisions. Furthermore, a complete set of pathologic data is only available for patients with resected cancers, which comprise a minority of patients with PDA. 
Improved prognostic information is a priority of cancer research. First, accurate prognosis informs discussions between oncologists and patients about the natural history of pancreatic cancer. Second, the information can guide treatment decisions with implications for both quality of life and cancer-related outcomes. The most biologically aggressive PDAs (such as those that recur soon after resection) are best treated initially with systemic therapy, as opposed to major surgery. Pancreatic surgery delays systemic treatment by a minimum of 2 months and exposes the patient to substantial operative risk with little expected benefit. On the other hand, patients with indolent cancers with oligometastatic disease may benefit from an aggressive surgical approach, as has become standard of care in selected patients with metastatic colorectal cancer [13]. Third, prognostic biomarkers provide mechanistic insights into cancer development. Fourth, they serve as molecular targets for novel treatment strategies such as vaccine [14], antibody [15], and promoter-driven gene therapies [16].

High impact studies based on hundreds of patient samples have improved prognostic capabilities in multiple cancer types (e.g. lung, prostate, colon, and breast) [17-20]. Studies of similar magnitude and scope have proven difficult in pancreatic cancer due to less available tissue for study and less biological heterogeneity between tumors. Perhaps the most informative prognostic study to date in PDA identified a panel of 6 prognostic markers based on gene expression differences between localized PDA and autopsy specimens $(\mathrm{n}=30)$ [21]. The rationale behind the study design was that the two study groups represented different ends of PDA extremes. In fact, the groups were actually distinguished by disease stage (i.e. early vs late), as opposed to biologic behavior (i.e. aggressive vs indolent). The localized group actually had a median survival of just 9 months, which is considered a short survival period post-resection [6].

In the present study, we used immunohistochemistry to interrogate a dichotomous set of resected PDAs $(n=137)$ comprised exclusively of aggressive (cancer-specific survival $<12$ months) and relatively less aggressive (cancer-specific survival $>30$ months) cancers, for true predictors of survival. A panel of 13 promising PDA biomarkers was selected from literally thousands of published PDA candidate biomarkers using a rigorous selection strategy (described in detail below), from on a public compendium of PDA biomarkers (Figure 1) [22]. Using this approach, we discounted 11 putative PDA biomarkers as prognostic markers. However, two proteins, mesothelin (MSLN) and mucin 1, cell surface associated (MUC1), were robust predictors of survival group and surpassed conventional pathologic features as prognostic factors. In this study, we demonstrated the utility of a largescale, high throughput immunohistochemistry (IHC) based-assay of PDAs at survival extremes to identify bona fide biomarkers of aggressive cancer biology.

\section{Methods}

\section{Patients}

This study was approved by the Memorial Sloan-Kettering Cancer Center (MSKCG) institution review board. Patients were included if they underwent a pancreatic resection for invasive tubular type (conventional) ductal adenocarcinoma (PDA) after the year 2000, and either died of disease within 1 year of resection (short survival) or survived at least 30 months (long survival). The specific survival boundaries were chosen for two reasons: first, to yield groups that were sufficiently powered for the analysis, yet had comparable sample sizes to each other; second, so that that the time interval between the two survival groups (at least 1.5 years in

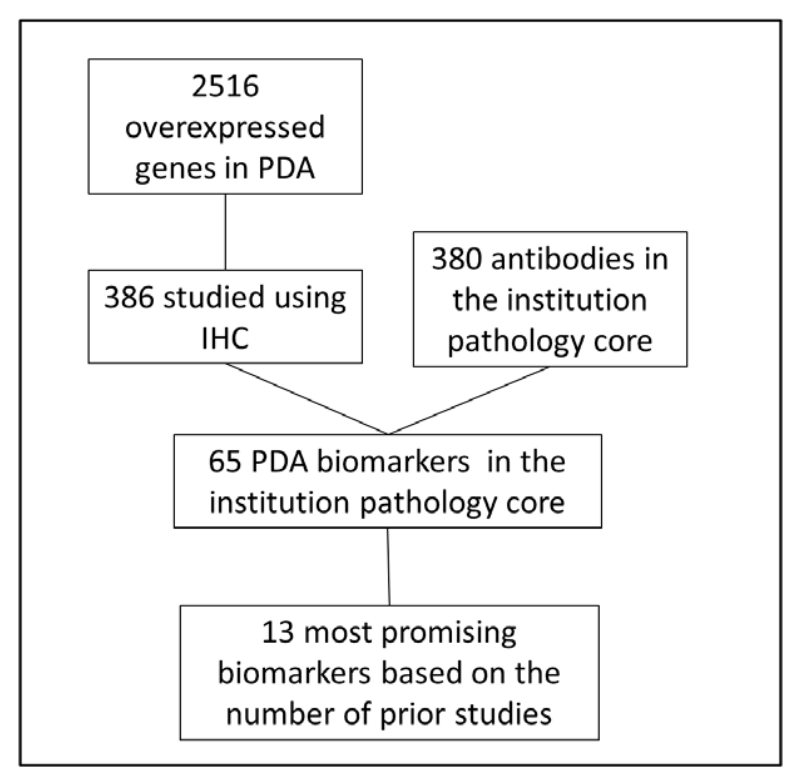

Figure 1. Algorithm for the selection of candidate biomarkers from a large public dataset of pancreatic cancer biomarkers [22].

doi:10.1371/journal.pone.0040157.g001

the present study) emphasized tumor biology over treatment related determinants of survival. For instance, adjuvant treatment provides a survival benefit of roughly 3 months for PDA [23], and therefore should not dictate survival groups as defined here, except in rare cases. Similarly, recovery rates from surgery are variable, but patients who survive pancreatic resection generally return to their preoperative baseline, or suffer from disease-related symptoms [24]. The records of each patient in the short-term survival group were meticulously reviewed, and only patients who died from pancreatic cancer (and not complications from surgery) were included in the study. Patients with invasive cancer arising from an intraductal papillary mucinous neoplasm, colloid carcinoma, acinar cell carcinoma, and other less common variants of adenocarcinoma were excluded.

\section{Clinicopathologic Information}

Clinicopathologic information was extracted from the institutional pancreatic tumor database and from electronic patient records. Relevant clinical variables included postoperative chemotherapy, radiation therapy, and patient survival. Pathologic data included lymph node status (positive vs. negative), tumor differentiation (poor vs. moderate/well), size ( $\geq 3 \mathrm{~cm}$ vs. $<3 \mathrm{~cm}$ ), and resection margin status (positive vs. negative). Microscopic disease at the pancreatic neck, bile duct, duodenum, and uncinate margins were categorized as positive.

\section{Tissue Preparation}

The TMA was constructed from tissue cores obtained from formalin-fixed, paraffin embedded tissue blocks in 151 patient samples. In all cases, tissue samples were derived from resected primary ductal adenocarcinomas of the pancreas. The technician placed the samples on the TMA in a blinded fashion ensuring that IHC interpretation by the study investigators was unbiased. The TMA was constructed as follows: a representative block of tumor was obtained and a corresponding $\mathrm{H} \& \mathrm{E}$ stained slide was examined under a microscope for foci of high neoplastic cellularity. Triplicate cores were taken from the index blocks 
Table 1. Clinicopathologic features in short- ( $<12$ months) and long-term survivors ( $>30$ months).

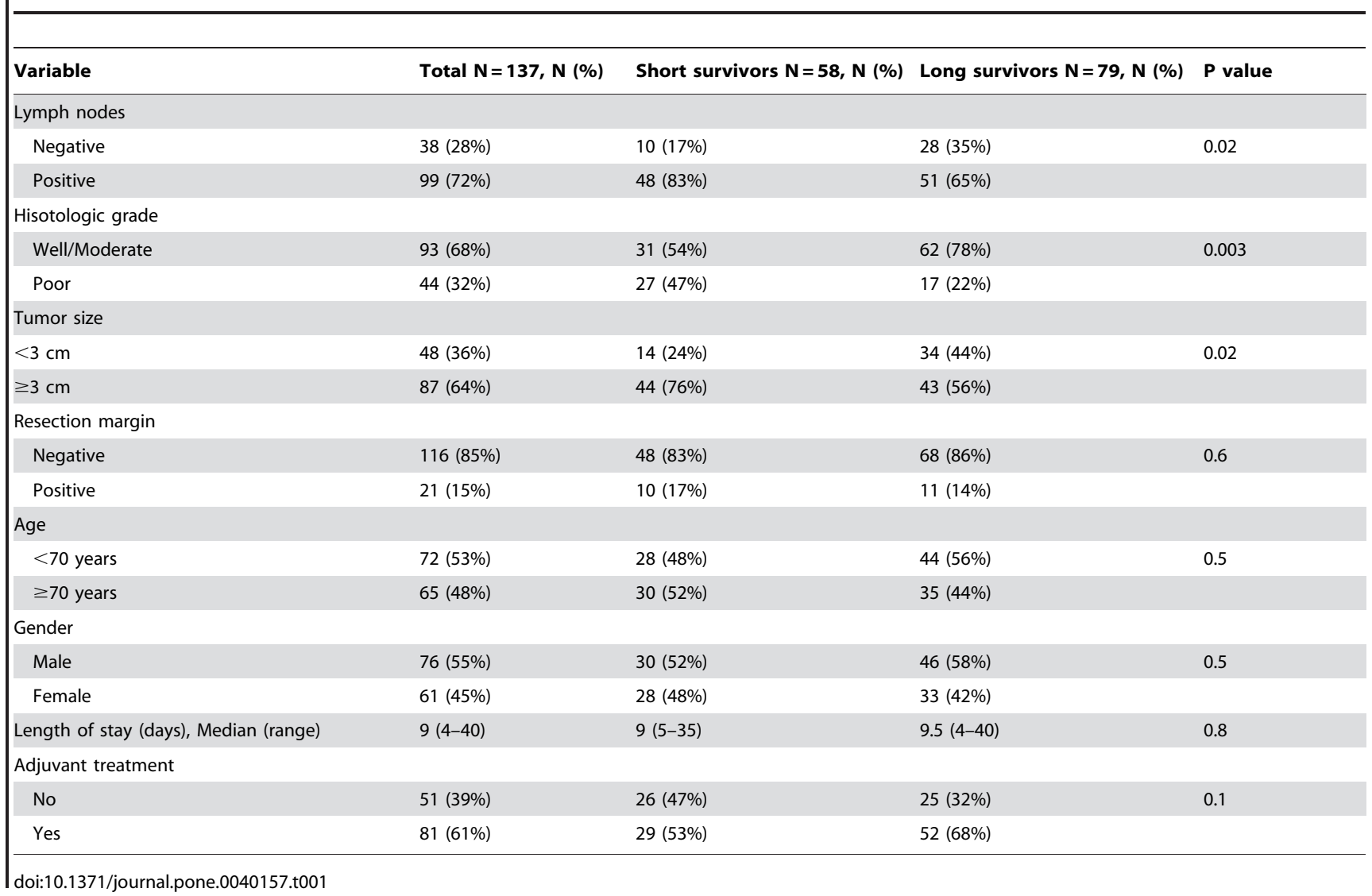

and transferred to a virgin block for TMA processing with an automated tissue array machine (ATA-27, Beecher Instruments, Silver Spring, MD). TMA sections were then cut from the block in preparation for immunohistochemistry experiments.

\section{Selection of Biomarkers}

A published compendium of putative pancreatic cancer biomarkers based on a comprehensive literature search lists 2,516 overexpressed genes $(\sim 10 \%$ of the genome) in pancreatic cancer [22]. Due to the overwhelming number of candidate biomarkers, we designed a strategy to select a panel of antibodies for use against the s-TMA (Figure 1). We pared the list of possibilities down to 386 candidate genes previously studied using IHC. The list of IHC biomarkers in PDA was then crossreferenced with our institutional pathology catalogue of 380 optimized antibodies. Our pathology core contained 65 optimized antibodies against putative PDA biomarkers. Due to the finite number of unstained histologic sections available from a TMA for study ( $\sim 50$ in the present resource), we further refined the selection process by stratifying biomarkers according to the number of independently published IHC studies cited in the central biomarker repository. Using this strategy, 13 PDA biomarkers were identified as the subject of four or more IHCbased peer-reviewed studies, and additionally were already optimized in our pathology core (BCL2, CASP3, CGND1, EGFR, ERBB2, MSLN, MUC1, MUC4, P53,SMAD4, MUC5AC, BIRC5, and ITGB4). The first 10 were selected for testing in the present study. In addition, three antibodies were included as representative samples from the remaining choices of putative PDA biomarkers: CEACAM6 (2 previous IHC-based publica- tions), MYC (2 publications), and MUC2 (1 publication). MUC2 was believed to be particularly intriguing due to its association with indolent pancreatic tumors, in direct contrast to MUC1, which has been linked with more aggressive pancreatic tumor subtypes $[25,26]$.

\section{Immunohistochemical Analysis}

Immunohistochemical analyses were performed by a standard streptavidin-biotin-peroxidase procedure. Labeled TMA sections were subjected to heat-induced epitope retrieval with the Ventana Discovery XT automated system (Ventana Medical Systems, Tucson, AZ). Primary antibodies and their dilutions included: BCL2 (1:100, DAKO, Carpenteria, CA), CASP3 (1:300), CCND1 (1:25, Lab Vision, Fremont, CA), CEACAM6 (1:5, Biogenex, San Ramon, CA), EGFR (1:100, Zymed, Carlsbad, CA), ERBB2 (1:400, Signet, Princeton, NJ), MSLN (1:100, Vector Labs, Burlingame, CA), MUC1 (1:100, Vector Labs, Burlingame, CA) [27], MUC2 (1:100 Vector Labs, Burlingame, CA) [27], MUC4 (1:3000, clone 8G7, a gift from University of Nebraska) [28], MYC (1:2000, Epitomics, Burlingame, CA), P53 (1:500, DAKO, Carpenteria, CA), and SMAD4 (1:800, Santa Cruz Bio, Santa Cruz, CA). The Ventana DABMap Kit was used for antibody detection.

Immunohistochemical review was performed by an expert pancreatic pathologist (L.H.T.) and recorded by a different study investigator (J.M.W). SMAD4, MUC2, and BCL2 were scored as negative or positive by IHC based on previous scoring strategies [29]. For all other antibodies, a 4-point scale (from 0 to $3+$ ) was applied based on the percentage of labeled cancer cells in the tissue core: $0(<10 \%$ labeled cells), $1+(11-25 \%), 2+(26 \%-75 \%), 3+$ 
Table 2. IHC analysis in short- ( $<12$ months) and long-term survivors ( $>30$ months).

\begin{tabular}{|c|c|c|c|c|c|}
\hline Biomarker, symbol & IHC Score & $\begin{array}{l}\text { Total } \mathbf{N}=137, \\
\text { N (\%) }\end{array}$ & $\begin{array}{l}\text { Short survivors } \\
\mathrm{N}=58, \mathrm{~N}(\%)\end{array}$ & $\begin{array}{l}\text { Long survivors } \\
\mathrm{N}=79, \mathrm{~N}(\%)\end{array}$ & $P$ value \\
\hline BCL2 & Negative & $137(100 \%)$ & $58(100 \%)$ & $79(100 \%)$ & - \\
\hline \multirow[t]{4}{*}{ CASP3 } & 0 & 85 (62\%) & 35 (60\%) & $50(63 \%)$ & 0.67 \\
\hline & $1+$ & $32(23 \%)$ & $16(28 \%)$ & $16(20 \%)$ & \\
\hline & $2+$ & $18(13 \%)$ & $6(10 \%)$ & $12(15 \%)$ & \\
\hline & $3+$ & $2(1 \%)$ & $1(2 \%)$ & $1(1 \%)$ & \\
\hline \multirow[t]{4}{*}{ CCND1 } & 0 & $17(12 \%)$ & $8(14 \%)$ & $9(11 \%)$ & 0.43 \\
\hline & $1+$ & $51(37 \%)$ & $21(36 \%)$ & $30(38 \%)$ & \\
\hline & $2+$ & $60(44 \%)$ & $23(40 \%)$ & $37(47 \%)$ & \\
\hline & $3+$ & $9(7 \%)$ & $6(11 \%)$ & $3(4 \%)$ & \\
\hline \multirow[t]{4}{*}{ CEACAM6 } & 0 & $14(10 \%)$ & $7(12 \%)$ & $7(9 \%)$ & 0.69 \\
\hline & $1+$ & $8(6 \%)$ & $2(3 \%)$ & $6(8 \%)$ & \\
\hline & $2+$ & $12(9 \%)$ & $6(10 \%)$ & $6(8 \%)$ & \\
\hline & $3+$ & $103(75 \%)$ & $43(74 \%)$ & $60(76 \%)$ & \\
\hline \multirow[t]{4}{*}{ EGFR } & 0 & 56 (41\%) & $24(41 \%)$ & $32(41 \%)$ & 1.0 \\
\hline & $1+$ & $38(28 \%)$ & $16(28 \%)$ & $22(29 \%)$ & \\
\hline & $2+$ & $38(28 \%)$ & $16(28 \%)$ & $22(29 \%)$ & \\
\hline & $3+$ & $5(4 \%)$ & $2(3 \%)$ & $3(4 \%)$ & \\
\hline \multirow[t]{3}{*}{ ERBB2 } & 0 & $111(81 \%)$ & $50(86 \%)$ & $61(79 \%)$ & 0.26 \\
\hline & $1+$ & $23(17 \%)$ & $8(14 \%)$ & $15(19 \%)$ & \\
\hline & $2+$ & $3(2 \%)$ & $0(0)$ & $3(4 \%)$ & \\
\hline \multirow[t]{4}{*}{ MSLN } & 0 & $40(29 \%)$ & $8(14 \%)$ & $32(41 \%)$ & $<0.0001$ \\
\hline & $1+$ & $31(23 \%)$ & $11(19 \%)$ & $20(25 \%)$ & \\
\hline & $2+$ & $45(33 \%)$ & $22(38 \%)$ & $23(29 \%)$ & \\
\hline & $3+$ & $21(15 \%)$ & 17 (29\%) & $4(5)$ & \\
\hline \multirow[t]{4}{*}{ MUC1 } & 0 & $20(15 \%)$ & $1(2 \%)$ & $19(24 \%)$ & $<0.0001$ \\
\hline & $1+$ & $31(23 \%)$ & $9(16 \%)$ & $22(28 \%)$ & \\
\hline & $2+$ & $45(33 \%)$ & $20(34 \%)$ & $25(32 \%)$ & \\
\hline & $3+$ & $41(30 \%)$ & $28(48 \%)$ & $13(16 \%)$ & \\
\hline \multirow[t]{2}{*}{ MUC2 } & Negative & $116(85 \%)$ & $54(93 \%)$ & $62(78 \%)$ & 0.03 \\
\hline & Positive & $21(15 \%)$ & $4(7 \%)$ & $17(22 \%)$ & \\
\hline \multirow[t]{4}{*}{ MUC4 } & 0 & $62(45 \%)$ & $23(40 \%)$ & 39 (49\%) & 0.70 \\
\hline & $1+$ & $30(22 \%)$ & $14(24 \%)$ & $16(20 \%)$ & \\
\hline & $2+$ & $18(13 \%)$ & $9(16 \%)$ & $9(11 \%)$ & \\
\hline & $3+$ & $27(20 \%)$ & $12(21 \%)$ & $15(19 \%)$ & \\
\hline \multirow[t]{4}{*}{ MYC } & 0 & $51(37 \%)$ & $25(43 \%)$ & $26(33 \%)$ & 0.32 \\
\hline & $1+$ & 42 (31\%) & 19 (33\%) & $23(29 \%)$ & \\
\hline & $2+$ & $38(28 \%)$ & $13(22 \%)$ & $25(32 \%)$ & \\
\hline & $3+$ & $6(4 \%)$ & $1(2 \%)$ & $5(6 \%)$ & \\
\hline \multirow[t]{2}{*}{ SMAD4 } & Negative & $43(31 \%)$ & $23(40 \%)$ & $20(25 \%)$ & 0.09 \\
\hline & Positive & $94(69 \%)$ & $35(60 \%)$ & 59 (75\%) & \\
\hline \multirow[t]{4}{*}{ TP53 } & 0 & 57 (42\%) & 22 (38\%) & 35 (44\%) & 0.21 \\
\hline & $1+$ & 17 (12\%) & $5(9 \%)$ & 12 (15\%) & \\
\hline & $2+$ & $35(26 \%)$ & $20(34 \%)$ & 15 (19\%) & \\
\hline & $3+$ & $28(20 \%)$ & $11(19 \%)$ & $17(22 \%)$ & \\
\hline
\end{tabular}

Approved gene names are listed.

Percentages reflect the fraction in a given column.

B-Cell CLL/Lymphoma 2; Caspase 3; Cyclin D1; Carcinoembryonic antigen-related cell adhesion molecule 6 (non-specific cross reacting antigen); Epidermal Growth Factor Receptor; V-erb-b2 avian erythroblastic leukemia viral oncogene homolog 2; Mesothelin; Mucin 1, cell surface associated; Mucin 2, oligomeric mucus/gel-forming; Mucin 4, cell surface associated; V-myc avian myelocytomatosis viral oncogene homolog; Mothers against decapentaplegic, drosophila, homolog of, 4 ; Tumor protein p53.

doi:10.1371/journal.pone.0040157.t002 
A

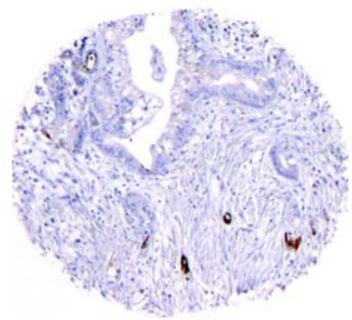

C

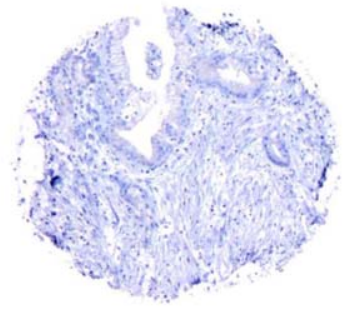

$\mathrm{E}$

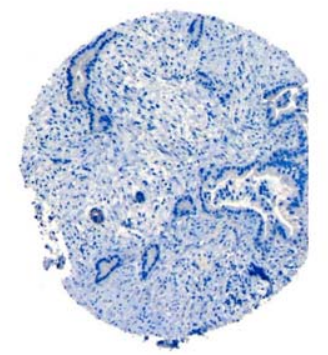

B

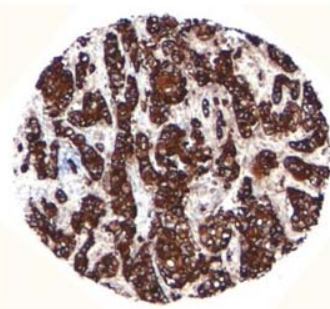

D

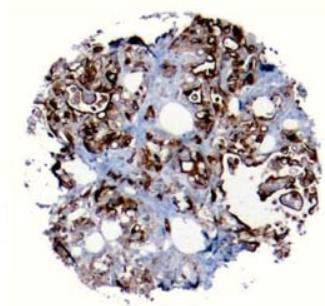

$\mathrm{F}$

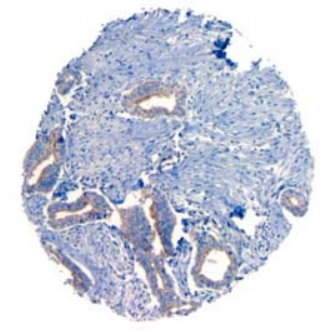

Figure 2. Representative immunolabeled slides: A) MUC1, 0; B) MUC1, 3+; C) MSLN, 0; D) MSLN, 3+; E) MUC2, negative; F) MUC2, positive.

doi:10.1371/journal.pone.0040157.g002

$(>75 \%)$. An average score was recorded for each triplicate set. A total of 14 samples had insufficient neoplastic cellularity for IHC analysis and were excluded, yielding 137 samples with adequate tissue for all tested antibodies.

\section{Statistical Analysis}

The analysis was performed using Intercooled Stata 8.2. Categorical variables were tested by the Fisher's exact test, continuous variables by the Wilcoxon rank sum test, and standard logistic regression was performed for multivariate testing. Continuous variables were tested using the rank sum test. A comparison of multivariate regression models was performed to identify the best prognostic model using receiver operating characteristic analysis and the associated Harrel's C-index (also referred to as area under the curve or ROC area). In the present analysis, the Cindex measures how well a particular multivariate model of predictors discriminates between short- and long-term survival groups. The values ranged between 0 and 1 . A value of 0.5 indicates no predictive ability (random prediction) and appears as a diagonal line on an ROC graph, whereas values above 0.5 indicate good predictability, and appear as curvilinear plots above the diagonal. When two ROC curves do not intersect, the one with a higher $\mathrm{C}$-index dominates over the other. All statistics were two-tailed with a $\mathrm{p}$ value $<0.05$ indicating statistical significance.

\section{Results}

\section{Conventional Pathologic Features}

There were $79(58 \%)$ patients in the long-term survivor group and $58(42 \%)$ in the short-term survivor group. Conventional pathologic features (lymph node status, histologic grade, size, and resection margin status) and patient variables (age, gender, postoperative length of stay, and adjuvant treatment) were analyzed as predictors of survival group (Table 1). None of the patient-related factors correlated with survival. Of the pathologic variables, positive lymph node status $(p=0.02)$, poor differentiation $(p=0.003)$, and a tumor size greater than $3 \mathrm{~cm}(p=0.02)$ were associated with early cancer-specific death in the unadjusted univariate analysis.

The prognostic accuracy of three different models, as estimated by the Harrell's C-index, was compared, graphed and tabulated (Figure 4). The model that included the three biomarkers (MUC1, MSLN, and MUC2) was superior to the model including four conventional pathologic features (lymph node status, histologic grade, tumor size, and resection margin status), although the difference just missed statistical significance $(p=0.07)$. The combined model with biomarkers and pathologic features performed the best $(p=0.0001)$.

\section{Biomarkers}

Expression patterns of the 13 candidate prognostic markers (see the Methods and Figure 1 for details on biomarker selection strategy) in the two survival groups were tested and compared. The univariate results are provided in Table 2. Out of 13 candidate genes, only MUC1, MSLN, and MUC2 had statistically different expression patterns between groups. A trend towards significance was observed with SMAD4 loss $(p=0.09)$. Representative slides labeled with MUC1, MSLN, and MUC2 appear in Figure 2.

\section{MUC1}

A strong association was observed between increased MUC1 protein expression and short survival $(\mathrm{p}<0.0001)$. In the total cohort, $15 \%$ of patients had an IHC score of $0,23 \%$ had $1+, 33 \%$ had $2+$, and $30 \%$ had $3+$. The proportions of patients that were in the short survivor group at each separate IHC score increased in a linear fashion (slope of linear regression $=0.21, p=0.002$ ). Specifically, $5 \%$ were in the short survival group with an IHC score of $0,29 \%$ with $1+, 49 \%$ with $2+$, and $68 \%$ with $3+$ (Figure 3A). The negative predictive value was high (95\%), as 19 out of 20 patients with absent MUC1 expression in this cohort survived more than 30 months.

\section{MSLN}

As compared to MUC1 expression, the pattern of MSLN expression in the total cohort was slightly weighted towards lower IHC scores: $63 \%$ of patients had 2+ or 3+ MUC1 labeling while $48 \%$ had comparable MSLN labeling $(\mathrm{p}=0.02)$. However, like MUC1, there was a strong association between MSLN expression and early cancer-specific mortality $(\mathrm{p}<0.0001)$. Again, a linear relationship was observed between the IHC score and the proportion of patients in the poor survival group (slope of linear regression $=0.20, p=0.02$ ). In the different IHC score categories, the percentage of patients that were in the short survival group were as follows: $20 \%$ of the patients with an IHC score of $0,35 \%$ with $1+, 49 \%$ with $2+$, and $81 \%$ with $3+$ (Figure $3 \mathrm{~B}$ ).

\section{MUC2}

MUC2 expression was associated with long survival in contrast to MUC1 and MSLN ( $\mathrm{p}=0.03)$. MUC2 expression was uncommon overall (15\%) in PDA. Short-term survivors expressed MUC2 in just $7 \%$ of cases. Long-term survivors expressed MUC2 in a 


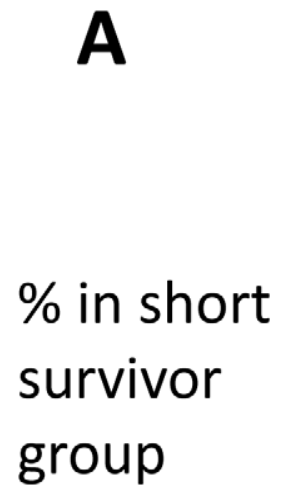

B
B in short
survivor
group

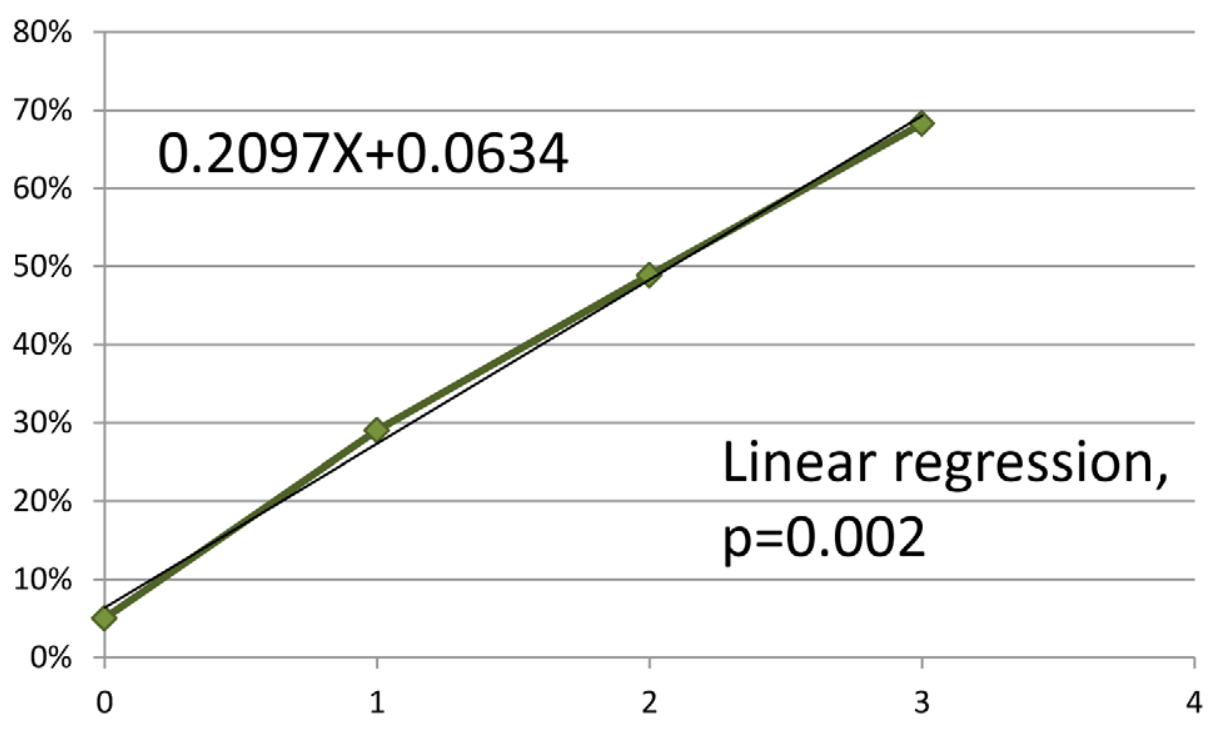

MUC1 IHC Score

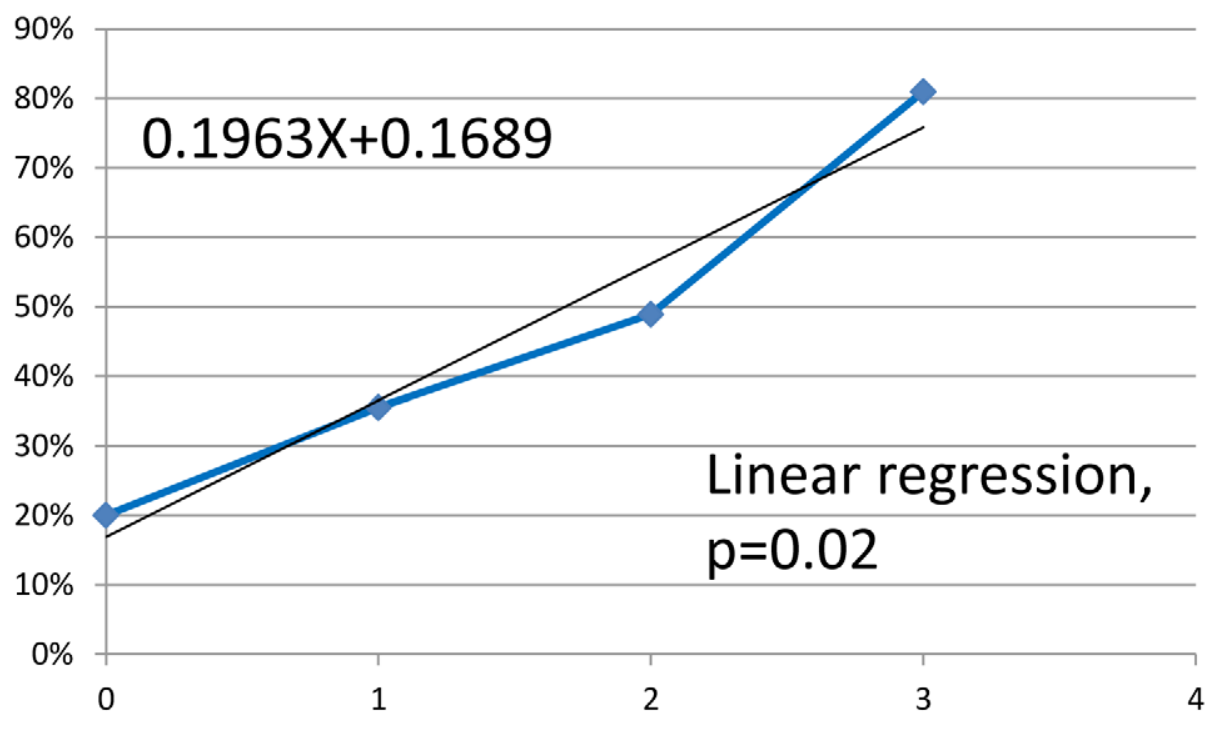

Mesothelin IHC Score

Figure 3. Short survivors (\% of total) vs IHC score: A) MUC1 B) MSLN. doi:10.1371/journal.pone.0040157.g003

greater proportion, although expression was still uncommon $(22 \%$ of cases).

\section{Multivariate Analysis}

A multivariate logistic regression analysis was performed which included significant univariate biomarker predictors of survival (MUC1, MSLN, and MUC2) as well as the four commonly reported pathologic features (Table 3). MUC1 and MSLN were highly significant in the adjusted model, while MUC2 was not. As compared to absent expression, odds ratios associated with incremental MSLN expression were 1.7 (IHC score $=1+$ ), 2.6 (IHC score $=2+$ ) and 12.5 (IHC score $=3+$ ). For MUC1, the odds ratios were 10.1 (IHC score $=1+$ ), 11.9 (IHC score $=2+$ ) and 29.0 (IHC score $=3+$ ). The composite p-values for MSLN and MUC1 were $p=0.01$ and $p=0.004$, respectively. None of the conventional pathologic features were statistically significant in the multivariate model. To test whether the high prognostic values of MUC1 and MSLN were merely an artifact of a multi-tiered comparison (IHC scores of 0 to $3+$ ), the multivariate model was repeated after categorizing lymph node metastases in a similarly tiered fashion (negative, 1, or $\geq 2$ lymph node metastases). Adjusted odds ratios for the relevant biomarkers were unchanged; multiple lymph node metastases predicted poor survival with an odds ratio of only $3.9(p=0.02)$. 
Table 3. Multivariate predictors of short-term survival.

\begin{tabular}{llll}
\hline & OR & 95\% CI & P value \\
\hline Prognostic marker & Ref & & 0.01 \\
\hline MSLN negative & 1.65 & $(0.48,5.72)$ & \\
MSLN 1+ & 2.64 & $(0.85,8.22)$ & \\
MSLN 2+ & 12.47 & $(2.43,64.14)$ & \\
MSLN 3+ & Ref & & 0.72 \\
MUC2 negative & 0.77 & $(0.18,3.32)$ & \\
MUC2 1+ & Ref & & 0.004 \\
MUC1 negative & 10.12 & $(1.05,97.50)$ & \\
MUC1 1+ & 11.91 & $(1.30,108.91)$ & \\
MUC1 2+ & 28.95 & $(2.93,285.64)$ & \\
MUC1 3+ & 2.79 & $(1.0,7.83)$ & 0.051 \\
\hline Positive lymph node & 2.22 & $(0.84,5.88)$ & 0.11 \\
Poor differentiation & 2.22 & $(0.89,5.52)$ & 0.09 \\
Size $\geq 3$ cm & 2.36 & $(0.71,7.85)$ & 0.16 \\
Positive resection margin & & & \\
\hline doi:10.1371/journal.pone.0040157.t003 & & & \\
\hline
\end{tabular}

\section{Discussion}

Early cancer recurrence and mortality after pancreatic resection (within one year) remain disheartening experiences for clinicians. In these instances, patients with seemingly "resectable" disease have major resections with "curative intent," yet do not receive any definable benefit, occasionally at the cost of significant morbidity or even mortality. Our institutional data suggests this scenario occurs in one-fifth of patients who undergo pancreatic resection for PDA [30]. On the other hand, some patients with metastatic disease have relatively slow growing cancers, and might benefit from metastasectomy or cytologic reduction. This scenario is extremely uncommon with PDA, yet there is precedent for an aggressive surgical approach in selected patients with advanced but indolent disease [31]. Unfortunately, the present approach to patients with PDA fails to integrate biologic factors. At the present time, conventional pathologic features provide the best prognostic information, yet are not sufficiently reliable to impact treatment decisions, as the present study shows.

Studies designed to identify reliable prognostic markers face two particular challenges. First, extrinsic determinants of survival which are independent of a tumor's molecular profile confound biomarker analyses. Consider a scenario in which the difference in overall survival between two patients after pancreatic resection is only 3 months. The survival difference may be related to patient performance status, social factors, medical comorbidities, chemotherapy response, treatment toxicity, surgical complications, or a number of other possibilities. Each of these factors may minimally contribute to patient survival, and would require a study with very large statistical power to fully characterize each one. Furthermore, these survival factors are not typically associated with biomarker expression patterns (chemotherapy response and toxicity may be exceptions). The present study minimizes noise from alternative and less significant survival factors by excluding patients with intermediate survival (12-30 months). Except for rare instances, tumor biology would be expected to be the principal driver of survival groups defined by a time gap of this magnitude (a minimum of 1.5 years separates short and long survivor groups).

We identified 20 other studies in the literature that analyzed protein biomarkers using TMAs of PDA (Table 4). Unlike the

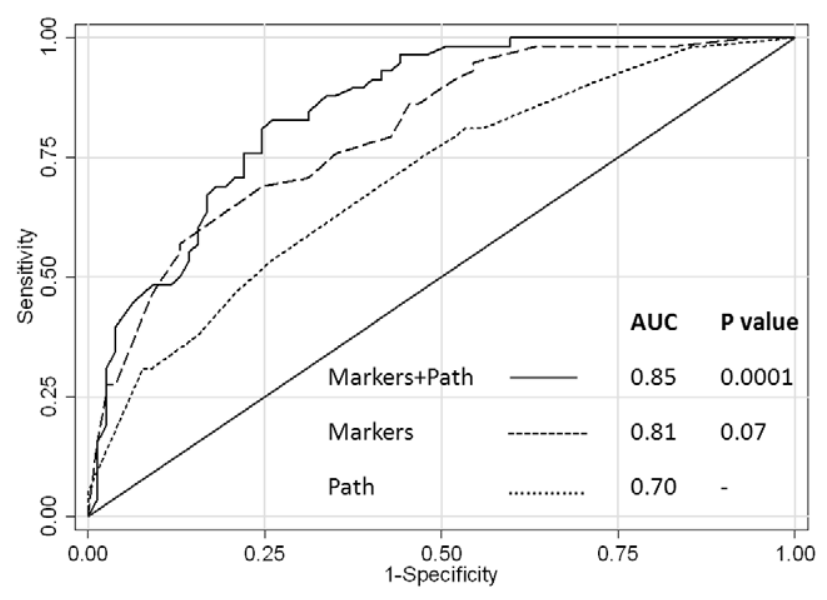

Figure 4. ROC curves of 3 predictive models of survival for the study cohort: Protein biomarkers and conventional pathologic features (- , MUC1, MUC2, MSLN, lymph node status, resection margin status, tumor differentiation); protein biomarkers only (----, MUC1, MUC2, MSLN); conventional pathologic features only (......, lymph node status, resection margin status, tumor differentiation, size). Values along the indicated diagonal line (line of no-discrimination) reflect a random guess, with points above the line being better than random. Harrel's C-index or area under the curve (AUC) for each plot is provided. $P$ values refer to comparisons between the given ROC curve as compared to pathologic features only.

doi:10.1371/journal.pone.0040157.g004

present study, these TMA-based studies included all patients across the survival spectrum, which may be interpreted as a positive study feature. None of these studies identified any biomarkers with clinical relevance in PDA. We suggest that a survival TMA may be better suited for biomarker discovery investigations in PDA, because it emphasizes tumor biology. The sample size in the present study compares favorably with other TMA studies (top quartile). Most important, this study likely includes the largest number of patients at the survival extremes.

The second challenge for biomarker surveys of PDA with IHC is to devise a rational strategy to select the best molecular candidates for study. There are roughly 30,000 human protein$\mathrm{s}$, and $10 \%$ have been reported as overexpressed in PDA [22]. IHC analyses are limited by the amount of available tissue (one antibody per TMA section, and roughly $30-50$ sections per TMA block), and therefore a rational candidate biomarker selection process is required to select the most practical and promising biomarkers for study. Typically, investigators design experimental biomarker panels according to either research interests, an intriguing paper, or a unifying theme such as a common molecular pathway. As Table 4 illustrates, previous TMA studies in PDA test a small number of antibodies (median of 2 biomarkers per study; range, 1 to 18). Only two studies examined more than 4 antibodies.

Biomarker selection in the present study was based on the recently published and centralized biomarker repository for PDA [22]. Construction of this dataset was a massive effort which required 7000 person hours (amounting to nearly one person's work per year). The authors identified every study in the literature that linked a gene or protein to PDA, and then tabulated the index gene (or protein), the principal assays involved in the study, and the relevant reference. The authors' primary goal was to "develop a compendium of potential biomarkers that could be systematically validated by the pancreatic cancer community" [22]. Putting their challenge to action, we analyzed this large dataset using an 
Table 4. Published tissue microarrays with pancreatic cancer.

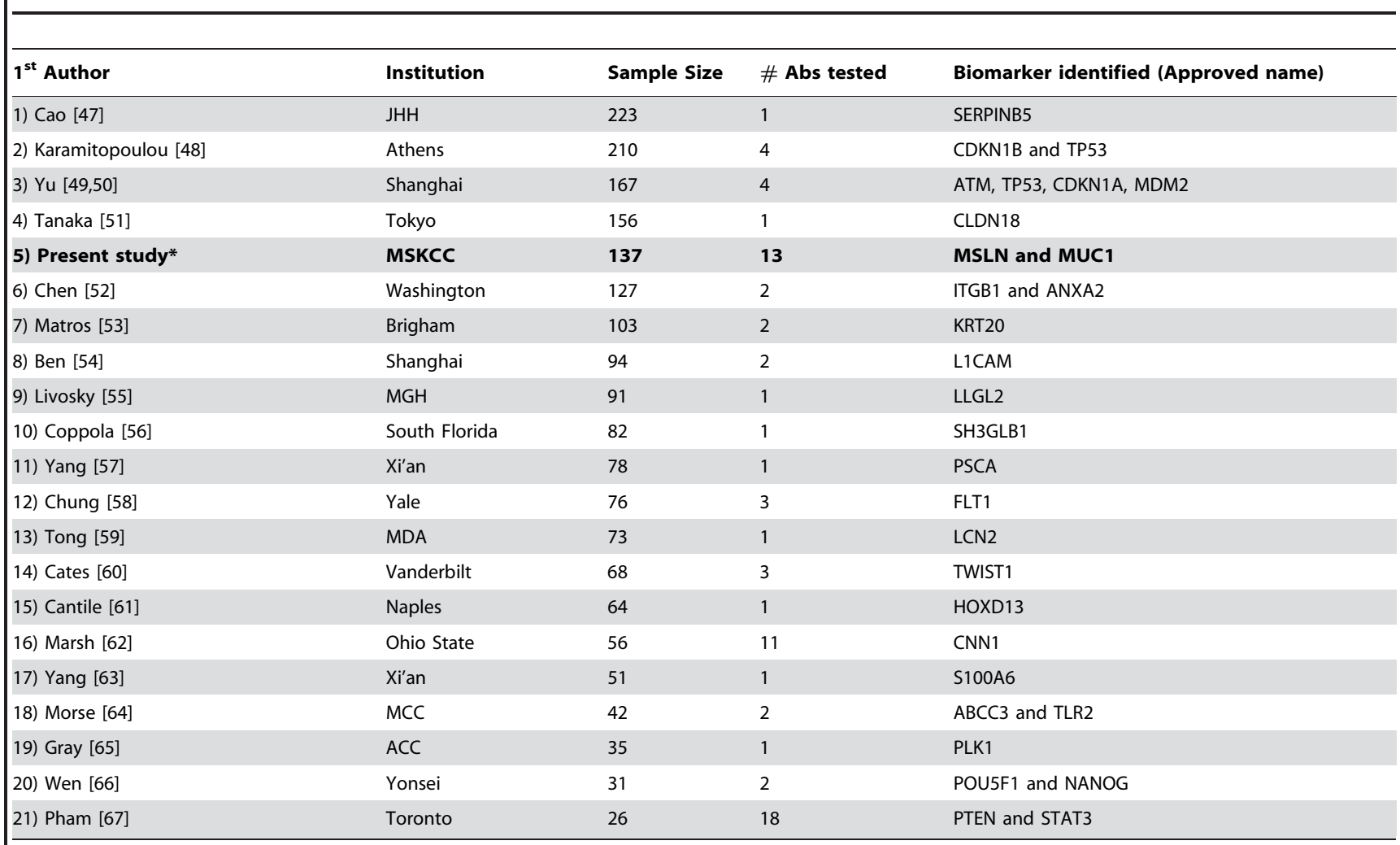

Pubmed search: tissue[Title/Abstract] AND microarray[Title/Abstract] AND pancreas and cancer.

Abbs: JHH (Johns Hopkins Hospital), MDA (MD Anderson), MCC (Moffitt Cancer Center), ACC (Arizona Cancer Center), Abs (antibodies).

*The present study is the only one that compared patients with short and long survival.

doi:10.1371/journal.pone.0040157.t004

algorithm (detailed in Figure 1) [22] that placed increased importance or weight on the number of previous reports linking a given biomarker to PDA. A total of 13 biomarkers were identified using this selection strategy and corresponding antibodies were tested against the s-TMA.

IHC analysis revealed that 10 of the 13 candidates were noninformative as prognostic markers in this study cohort. These negative observations provide convincing evidence (with the exception of SMAD4 which missed statistical significance, $\mathrm{p}=0.09$ ) that this group of putative pancreatic cancer biomarkers are clinically irrelevant for prognosis. In the univariate analysis, MUC1 and MSLN expression were associated with aggressive cancer biology (i.e. short survival group) and MUC2 expression was associated with favorable biology (i.e. long survival group). Only MUC1 and MSLN were robust prognostic factors in the multivariate model, adjusting for conventional pathologic features (Table 3). Diffuse MUC1 and MSLN expression were highly predictive of short survival (the odds ratios were 12.47 and 28.95, respectively). Interestingly, the four standard pathology tests had odds ratios below 3, and none achieved statistical significance in the multivariate model. An ROC analysis was performed to estimate the predictive accuracy of three different multivariate models at distinguishing short and long survival groups (biomarkers only; pathologic features only; and a combination of biomarkers and pathologic features). A trend towards superior predictive accuracy was observed with the panel of molecular markers (MUC1, MSLN, and MUC2) over conventional pathologic features $(\mathrm{AUC}=0.81$ vs. $0.70, \mathrm{p}=0.07)$. These data suggest that biomarkers may actually provide more prognostic insight than standard prognostic data included in pathology reports.

This study validates MUC1 and MSLN as biomarkers of aggressive pancreatic cancer biology. The implications of these findings must be interpreted in the context of the study design, and the role of these proteins as prognostic markers in the clinical management of PDA remains uncertain. While each oncoprotein has been the focus of over 200 studies in PDA, there are no largescale studies of tumor samples that have thoroughly examined them as prognostic biomarkers using a comparable graded IHC scoring system. Some previous studies have observed survival differences associated with high and low expressing tumors (MUC1 or MSLN), but are limited by small sample sizes, the absence of tiered IHC scoring systems, and unadjusted statistics [32-34].

Certain biomarkers included in the study had expression patterns that differed from previous reports. For instance, MSLN expression (1+ or greater) was observed in $71 \%$ of patients with PDA in the present study (as compared to $85-100 \%$ in prior studies [32,35]) and MUC4 expression (1+ or greater) was observed in $55 \%$ (as compared to $90 \%$ in prior studies $[36,37]$ ). Differences between this study and previous ones may be related to sample size variability (previous studies were smaller) and patient selection (the present study is enriched with patients at the survival extremes). We are in the process of validating the results of the present study in a large dataset that includes patients across the entire survival spectrum. In addition, differences in immunohistochemical scoring are important. For instance, previous studies of MSLN and MUC4 defined positive labeling as focal antibody 
reactivity in more than $1 \%$ of cancer cells [35,36], while the present study required at least $10 \%$ of cells for an IHC score of $1+$.

The implications of this research extend beyond improved prognostic assessment of tumor samples, and therefore the utility of the s-TMA strategy is not entirely contingent on validation studies with large numbers of unselected patients. First, biomarker discovery based on survival extremes is useful to prioritize cancer genes for diagnostic and therapeutic research. Since the most biologically aggressive cancer cells are typically refractory to conventional agents, it stands to reason that novel treatment approaches that specifically target aggressive sub-clones are particularly appealing and warrant further investigation. In support of this concept, the NCI has identified MUCl and MSLN among the most promising targets for cancer vaccine development, with the former protein listed in the top three [38]. A radiolabeled monoclonal antibody against MUC1 was also recently evaluated in a phase I/II trial, with a planned phase III trial to follow $[39,40]$. Furthermore, promoter-driven cancer gene therapy which exploits overactive MUC1 and MSLN promoters in various cancer types has been extensively studied in pre-clinical cancer models using viral vectors [41-43]. We are currently pursuing a promoter-driven gene therapy approach against PDA using a non-viral, biodegradable polymer vector to deliver toxic nanoparticles [16].

Additionally, both MUC1 and MSLN are present on the cellsurface with secreted isoforms. Thus, prognostic markers such as these are potentially detectable in sera or secreted fluids. Reliable noninvasive tests that correlate with membrane-bound isoforms may function as surrogate biomarkers to biologically stratify patients or perhaps select them for targeted therapies. An FDA approved ELISA test of soluble mesothelin-related proteins (Mesomark ${ }^{\circledR}$ Assay, Fujirebio Diagnostics, Malvern, PA) holds great promise as a serum and pleural fluid marker for malignant pleural mesothelioma [44]. The Mesomark Assay has been evaluated in a single study of PD; the study was not powered to test prognostic capability and did not compare levels with tumor MSLN expression [45]. No studies have examined the prognostic potential of soluble-MUC1 in PDA.

We are presently evaluating additional candidate prognostic markers against the s-TMA to further optimize the predictive

\section{References}

1. Kalser MH, Ellenberg SS (1985) Pancreatic cancer. Adjuvant combined radiation and chemotherapy following curative resection. Arch Surg 120: 899-903.

2. Neoptolemos JP, Stocken DD, Friess H, Bassi C, Dunn JA, et al. (2004) A randomized trial of chemoradiotherapy and chemotherapy after resection of pancreatic cancer. N Engl J Med 350: 1200-1210.

3. Oettle H, Post S, Neuhaus P, Gellert K, Langrehr J, et al. (2007) Adjuvant chemotherapy with gemcitabine vs observation in patients undergoing curativeintent resection of pancreatic cancer: a randomized controlled trial. JAMA 297: 267-277.

4. Regine WF, Winter KA, Abrams RA, Safran H, Hoffman JP, et al. (2008) Fluorouracil vs gemcitabine chemotherapy before and after fluorouracil-based chemoradiation following resection of pancreatic adenocarcinoma: a randomized controlled trial. JAMA 299: 1019-1026.

5. Jones S, Zhang X, Parsons DW, Lin JC, Leary RJ, et al. (2008) Core signaling pathways in human pancreatic cancers revealed by global genomic analyses. Science 321: 1801-1806.

6. Winter JM, Cameron JL, Campbell KA, Arnold MA, Chang DC, et al. (2006) 1423 pancreaticoduodenectomies for pancreatic cancer: A single-institution experience. J Gastrointest Surg 10: 1199-1210; discussion 1210-1191.

7. Berger AC, Garcia M Jr, Hoffman JP, Regine WF, Abrams RA, et al. (2008) Postresection CA 19-9 predicts overall survival in patients with pancreatic cancer treated with adjuvant chemoradiation: a prospective validation by RTOG 9704. J Clin Oncol 26: 5918-5922.

8. Garcea G, Dennison AR, Pattenden CJ, Neal CP, Sutton CD, et al. (2008) Survival following curative resection for pancreatic ductal adenocarcinoma. A systematic review of the literature. JOP 9: 99-132. model. Based on the strategy used to select candidate markers in the present study, additional intriguing proteins include MUC5AC, BIRC5, and ITGB4. A high-throughput proteomic or transcriptomic analysis of survival extremes could identify novel prognostic markers, but is best suited for tumor samples enriched for neoplastic cells such as tumor cell lines or xenografts (as opposed to primary tumor tissue such as the samples in this study with abundant stroma) [46]. A high-throughput molecular analytic strategy would obviate the need for a pre-assay biomarker selection process for candidate immunohistochemical markers such as the one described in Figure 1. The disadvantage is that the results reflect the molecular profile of a clonal cancer cell population derived from the original tumor (and likely a particularly aggressive clone selected for under laboratory conditions), which may not reflect the biology of the rest of the tumor. Gene expression analyses comparable to the Oncotype $\mathrm{Dx}^{\circledR}$ for breast cancer [18], would likely require very large numbers of primary tumor samples to determine an effective prognostic panel, particularly because of less biologic heterogeneity with PDA.

\section{Conclusions}

This study presents the results of a survival-based TMA (sTMA), comprised of patient tumor samples associated with short and long-term survival after resection for PDA. The s-TMA was used to identify bona fide protein markers of aggressive tumor biology. MSLN and MUCil were highly significant predictors of early cancer-specific mortality, and were superior to conventional pathologic features as prognostic markers.

\section{Acknowledgments}

We thank Irena Linkov for performing the immunohistochemistry.

\section{Author Contributions}

Conceived and designed the experiments: JMW LHT DSK FGR PJA. Performed the experiments: JMW LHT. Analyzed the data: JMW XJ LQ. Contributed reagents/materials/analysis tools: LHT DSK. Wrote the paper: JMW MFB JRB MID RPD YF WRJ EMO PJA.

9. Brennan MF, Kattan MW, Klimstra D, Conlon K (2004) Prognostic nomogram for patients undergoing resection for adenocarcinoma of the pancreas. Ann Surg 240: 293-298.

10. Kondo N, Murakami Y, Uemura K, Hayashidani Y, Sudo T, et al. (2010) Prognostic impact of perioperative serum CA 19-9 levels in patients with resectable pancreatic cancer. Ann Surg Oncol 17: 2321-2329.

11. Hartwig W, Hackert T, Hinz U, Gluth A, Bergmann F, et al. (2011) Pancreatic cancer surgery in the new millennium: better prediction of outcome. Ann Surg 254: $311-319$.

12. Barton JG, Bois JP, Sarr MG, Wood CM, Qin R, et al. (2009) Predictive and prognostic value of CA 19-9 in resected pancreatic adenocarcinoma. J Gastrointest Surg 13: 2050-2058.

13. Nordlinger B, Sorbye H, Glimelius B, Poston GJ, Schlag PM, et al. (2008) Perioperative chemotherapy with FOLFOX4 and surgery versus surgery alone for resectable liver metastases from colorectal cancer (EORTC Intergroup trial 40983): a randomised controlled trial. Lancet 371: 1007-1016.

14. Leao IC, Ganesan P, Armstrong TD, Jaffee EM (2008) Effective depletion of regulatory $\mathrm{T}$ cells allows the recruitment of mesothelin-specific CD8 T cells to the antitumor immune response against a mesothelin-expressing mouse pancreatic adenocarcinoma. Clin Transl Sci 1: 228-239.

15. Feng Y, Xiao X, Zhu Z, Streaker E, Ho M, et al. (2009) A novel human monoclonal antibody that binds with high affinity to mesothelin-expressing cells and kills them by antibody-dependent cell-mediated cytotoxicity. Mol Cancer Ther 8: 1113-1118.

16. Showalter SL, Huang YH, Witkiewicz A, Costantino CL, Yeo CJ, et al. (2008) Nanoparticulate delivery of diphtheria toxin DNA effectively kills Mesothelin expressing pancreatic cancer cells. Cancer Biol Ther 7: 1584-1590. 
17. Shedden K, Taylor JM, Enkemann SA, Tsao MS, Yeatman TJ, et al. (2008) Gene expression-based survival prediction in lung adenocarcinoma: a multi-site, blinded validation study. Nat Med 14: 822-827.

18. Paik S, Shak S, Tang G, Kim C, Baker J, et al. (2004) A multigene assay to predict recurrence of tamoxifen-treated, node-negative breast cancer. N Engl J Med 351: 2817-2826.

19. Gray RG, Quirke P, Handley K, Lopatin M, Magill L, et al. (2011) Validation study of a quantitative multigene reverse transcriptase-polymerase chain reaction assay for assessment of recurrence risk in patients with stage II colon cancer. J Clin Oncol 29: 4611-4619.

20. Cheville JC, Karnes RJ, Therneau TM, Kosari F, Munz JM, et al. (2008) Gene panel model predictive of outcome in men at high-risk of systemic progression and death from prostate cancer after radical retropubic prostatectomy. J Clin Oncol 26: 3930-3936.

21. Stratford JK, Bentrem DJ, Anderson JM, Fan C, Volmar KA, et al. (2010) A sixgene signature predicts survival of patients with localized pancreatic ductal adenocarcinoma. PLoS Med 7: e1000307.

22. Harsha HC, Kandasamy K, Ranganathan P, Rani S, Ramabadran S, et al. (2009) A compendium of potential biomarkers of pancreatic cancer. PLoS Med 6: e1000046.

23. Boeck S, Ankerst DP, Heinemann V (2007) The role of adjuvant chemotherapy for patients with resected pancreatic cancer: systematic review of randomized controlled trials and meta-analysis. Oncology 72: 314-321.

24. Huang JJ, Yeo CJ, Sohn TA, Lillemoe KD, Sauter PK, et al. (2000) Quality of life and outcomes after pancreaticoduodenectomy. Ann Surg 231: 890-898.

25. Shi C, Hruban RH (2012) Intraductal papillary mucinous neoplasm. Hum Pathol 43: 1-16.

26. Levi E, Klimstra DS, Andea A, Basturk O, Adsay NV (2004) MUC1 and MUC2 in pancreatic neoplasia. J Clin Pathol 57: 456-462.

27. Adsay NV, Merati K, Nassar H, Shia J, Sarkar F, et al. (2003) Pathogenesis of colloid (pure mucinous) carcinoma of exocrine organs: Coupling of gel-forming mucin (MUC2) production with altered cell polarity and abnormal cell-stroma interaction may be the key factor in the morphogenesis and indolent behavior of colloid carcinoma in the breast and pancreas. Am J Surg Pathol 27: 571-578.

28. Jain M, Venkatraman G, Moniaux N, Kaur S, Kumar S, et al. (2011) Monoclonal antibodies recognizing the non-tandem repeat regions of the human mucin MUC4 in pancreatic cancer. PLoS One 6: e23344.

29. Iacobuzio-Donahue CA, Fu B, Yachida S, Luo M, Abe H, et al. (2009) DPC4 gene status of the primary carcinoma correlates with patterns of failure in patients with pancreatic cancer. J Clin Oncol 27: 1806-1813.

30. Winter JM, Brennan MF, Tang LH, D'Angelica MI, Dematteo RP, et al. (2012) Survival after resection of pancreatic adenocarcinoma: results from a single institution over three decades. Ann Surg Oncol 19: 169-175.

31. Arnaoutakis GJ, Rangachari D, Laheru DA, Iacobuzio-Donahue CA, Hruban $\mathrm{RH}$, et al. (2011) Pulmonary resection for isolated pancreatic adenocarcinoma metastasis: an analysis of outcomes and survival. J Gastrointest Surg 15: 16111617.

32. Einama T, Kamachi H, Nishihara H, Homma S, Kanno H, et al. (2011) Coexpression of mesothelin and CA125 correlates with unfavorable patient outcome in pancreatic ductal adenocarcinoma. Pancreas 40: 1276-1282.

33. Hinoda Y, Ikematsu Y, Horinochi M, Sato S, Yamamoto K, et al. (2003) Increased expression of MUC1 in advanced pancreatic cancer. J Gastroenterol 38: 1162-1166.

34. Takikita M, Altekruse S, Lynch CF, Goodman MT, Hernandez BY, et al. (2009) Associations between selected biomarkers and prognosis in a population-based pancreatic cancer tissue microarray. Cancer Res 69: 2950-2955.

35. Argani P, Iacobuzio-Donahue C, Ryu B, Rosty C, Goggins M, et al. (2001) Mesothelin is overexpressed in the vast majority of ductal adenocarcinomas of the pancreas: identification of a new pancreatic cancer marker by serial analysis of gene expression (SAGE). Clin Cancer Res 7: 3862-3868.

36. Swartz MJ, Batra SK, Varshney GC, Hollingsworth MA, Yeo CJ, et al. (2002) MUC4 expression increases progressively in pancreatic intraepithelial neoplasia. Am J Clin Pathol 117: 791-796.

37. Jhala N, Jhala D, Vickers SM, Eltoum I, Batra SK, et al. (2006) Biomarkers in Diagnosis of pancreatic carcinoma in fine-needle aspirates. Am J Clin Pathol 126: $572-579$.

38. Cheever MA, Allison JP, Ferris AS, Finn OJ, Hastings BM, et al. (2009) The prioritization of cancer antigens: a national cancer institute pilot project for the acceleration of translational research. Clin Cancer Res 15: 5323-5337.

39. Wegener W (2008) Safety and Efficacy Study of 90Y-hPAM4 at Different Doses.

40. Cardillo TM, Ying Z, Gold DV (2001) Therapeutic advantage of (90)yttriumversus (131)iodine-labeled PAM4 antibody in experimental pancreatic cancer. Clin Cancer Res 7: 3186-3192.

41. Chen L, Pulsipher M, Chen D, Sieff C, Elias A, et al. (1996) Selective transgene expression for detection and elimination of contaminating carcinoma cells in hematopoietic stem cell sources. J Clin Invest 98: 2539-2548.

42. Kurihara T, Brough DE, Kovesdi I, Kufe DW (2000) Selectivity of a replicationcompetent adenovirus for human breast carcinoma cells expressing the MUC1 antigen. J Clin Invest 106: 763-771.
43. Tsuruta Y, Pereboeva L, Breidenbach M, Rein DT, Wang M, et al. (2008) A fiber-modified mesothelin promoter-based conditionally replicating adenovirus for treatment of ovarian cancer. Clin Cancer Res 14: 3582-3588.

44. Hollevoet K, Reitsma JB, Creaney J, Grigoriu BD, Robinson BW, et al. (2012) Serum mesothelin for diagnosing malignant pleural mesothelioma: an individual patient data meta-analysis. J Clin Oncol 30: 1541-1549.

45. Sharon E, Zhang J, Hollevoet K, Steinberg SM, Pastan I, et al. (2011) Serum mesothelin and megakaryocyte potentiating factor in pancreatic and biliary cancers. Clin Chem Lab Med.

46. Winter JM, Brody JR, Kern SE (2006) Multiple-criterion evaluation of reported mutations: a proposed scoring system for the intragenic somatic mutation literature. Cancer Biol Ther 5: 360-370.

47. Cao D, Zhang Q, Wu LS, Salaria SN, Winter JW, et al. (2007) Prognostic significance of maspin in pancreatic ductal adenocarcinoma: tissue microarray analysis of 223 surgically resected cases. Mod Pathol 20: 570-578.

48. Karamitopoulou E, Zlobec I, Tornillo L, Carafa V, Schaffner T, et al. (2010) Differential cell cycle and proliferation marker expression in ductal pancreatic adenocarcinoma and pancreatic intraepithelial neoplasia (PanIN). Pathology 42: 229-234.

49. Yu GZ, Zhu MH, Chen Y, Ni CR, Li FM (2005) [Expression and clinical significance of $\mathrm{P} 53$ pathway-associated proteins in pancreatic carcinoma]. Ai Zheng 24: 1398-1403.

50. Yu G, Zhu MH, Zhu Z, Ni CR, Zheng JM, et al. (2004) Expression of ATM protein and its relationship with p53 in pancreatic carcinoma with tissue array. Pancreas 28: 421-426.

51. Tanaka M, Shibahara J, Fukushima N, Shinozaki A, Umeda M, et al. (2011) Claudin-18 is an early-stage marker of pancreatic carcinogenesis. J Histochem Cytochem 59: 942-952.

52. Chen R, Yi EC, Donohoe S, Pan S, Eng J, et al. (2005) Pancreatic cancer proteome: the proteins that underlie invasion, metastasis, and immunologic escape. Gastroenterology 129: 1187-1197.

53. Matros E, Bailey G, Clancy T, Zinner M, Ashley S, et al. (2006) Cytokeratin 20 expression identifies a subtype of pancreatic adenocarcinoma with decreased overall survival. Cancer 106: 693-702.

54. Ben QW, Wang JC, Liu J, Zhu Y, Yuan F, et al. (2010) Positive expression of L1-CAM is associated with perineural invasion and poor outcome in pancreatic ductal adenocarcinoma. Ann Surg Oncol 17: 2213-2221.

55. Lisovsky M, Dresser K, Woda B, Mino-Kenudson M (2010) Immunohistochemistry for cell polarity protein lethal giant larvae 2 differentiates pancreatic intraepithelial neoplasia-3 and ductal adenocarcinoma of the pancreas from lower-grade pancreatic intraepithelial neoplasias. Hum Pathol 41: 902-909.

56. Coppola D, Helm J, Ghayouri M, Malafa MP, Wang HG (2011) Downregulation of Bax-interacting factor 1 in human pancreatic ductal adenocarcinoma. Pancreas 40: 433-437.

57. Yang WB, Cai F, Cheng CT, Cao G, Qing ZY (2009) [Role of prostate stem cell antigen in human pancreatic carcinoma: a tissue microarray-based study]. Nan Fang Yi Ke Da Xue Xue Bao 29: 2135-2137.

58. Chung GG, Yoon HH, Zerkowski MP, Ghosh S, Thomas L, et al. (2006) Vascular endothelial growth factor, FLT-1, and FLK-1 analysis in a pancreatic cancer tissue microarray. Cancer 106: 1677-1684.

59. Tong Z, Kunnumakkara AB, Wang H, Matsuo Y, Diagaradjane P, et al. (2008) Neutrophil gelatinase-associated lipocalin: a novel suppressor of invasion and angiogenesis in pancreatic cancer. Cancer Res 68: 6100-6108.

60. Cates JM, Byrd RH, Fohn LE, Tatsas AD, Washington MK, et al. (2009) Epithelial-mesenchymal transition markers in pancreatic ductal adenocarcinoma. Pancreas 38: e1-6.

61. Cantile M, Franco R, Tschan A, Baumhoer D, Zlobec I, et al. (2009) HOX D13 expression across 79 tumor tissue types. Int J Cancer 125: 1532-1541.

62. Marsh WL, Colonna J, Yearsley M, Bloomston M, Frankel WL (2009) Calponin is expressed in serous cystadenomas of the pancreas but not in adenocarcinomas or endocrine tumors. Appl Immunohistochem Mol Morphol 17: 216-219.

63. Yang WB, Wang YH, Cai F, Cao G, Qin ZY (2007) [Biological significance of expression of calcyclin in human pancreatic carcinoma: a tissue microarraybased study]. Nan Fang Yi Ke Da Xue Xue Bao 27: 1908-1910.

64. Morse DL, Balagurunathan Y, Hostetter G, Trissal M, Tafreshi NK, et al. (2010) Identification of novel pancreatic adenocarcinoma cell-surface targets by gene expression profiling and tissue microarray. Biochem Pharmacol 80: 748754.

65. Gray PJ Jr, Bearss DJ, Han H, Nagle R, Tsao MS, et al. (2004) Identification of human polo-like kinase 1 as a potential therapeutic target in pancreatic cancer. Mol Cancer Ther 3: 641-646.

66. Wen J, Park JY, Park KH, Chung HW, Bang S, et al. (2010) Oct4 and Nanog expression is associated with early stages of pancreatic carcinogenesis. Pancreas 39: 622-626.

67. Pham NA, Schwock J, Iakovlev V, Pond G, Hedley DW, et al. (2008) Immunohistochemical analysis of changes in signaling pathway activation downstream of growth factor receptors in pancreatic duct cell carcinogenesis. BMC Cancer 8: 43. 